\title{
Radiative transfer in decomposed domains ${ }^{\star}$
}

\author{
T. Heinemann ${ }^{1,2}$, W. Dobler ${ }^{3,4}, \AA$ A. Nordlund ${ }^{2}$, and A. Brandenburg ${ }^{1}$
}

\author{
1 NORDITA, Blegdamsvej 17, 2100 Copenhagen $\varnothing$, Denmark \\ e-mail: theine@nordita.dk \\ 2 Niels Bohr Institute, Copenhagen University, Juliane Maries Vej 30, 2100 Copenhagen Ø, Denmark \\ 3 Department of Physics and Astronomy, University of Calgary, Calgary, Alberta, Canada \\ 4 Kiepenheuer-Institut für Sonnenphysik, Schöneckstraße 6, 79104 Freiburg, Germany
}

Received 23 March 2005 / Accepted 7 November 2005

\section{ABSTRACT}

Aims. An efficient algorithm for calculating radiative transfer on massively parallel computers using domain decomposition is presented. Methods. The integral formulation of the transfer equation is used to divide the problem into a local but compute-intensive part for calculating the intensity and optical depth integrals, and a nonlocal part for communicating the intensity between adjacent processors.

Results. The waiting time of idle processors during the nonlocal communication part does not have a severe impact on the scaling. The wall clock time thus scales nearly linearly with the inverse number of processors.

Key words. radiative transfer - methods: numerical - hydrodynamics

\section{Introduction}

Over the past one or two decades tremendous advances have been made in achieving high resolution power in computational astrophysical fluid dynamics; see Haugen et al. (2003) for a $1024^{3}$ simulation of hydromagnetic turbulence and Kaneda et al. (2003) for a $4096^{3}$ simulation without magnetic fields. Such high resolution is now possible mainly due to the availability of massively parallel computers allowing superb performance at a low price, especially if off-the-shelf personal computers can be interconnected using standard Ethernet switches. This poses no major difficulty for the usual finite difference schemes that allow the computational domain to be decomposed into smaller sub-domains, because the necessary communication between processors is limited to a small neighborhood of the processor boundaries.

Radiative transfer calculations fall generally outside this class of problems, because the transfer equation is intrinsically nonlocal. Physically speaking, information travels at the speed of light when the gas is optically thin. Thus, from one time step to the next, the change in intensity in the domain of one processor can affect the radiation field on many other processors even if they are far apart.

In this paper we describe a simple method that renders the transfer problem essentially local - at least as far as the bulk of the computational cost is concerned. By using the integral formulation of the transfer equation, the intensity may be written

* Appendices are only available in electronic form at http://wWw.edpsciences.org as a local integral term plus an attenuated boundary term. The local integral term may be computed in parallel by all processors whereas only the boundary term, which may be applied after the integrals have been computed on all processors, requires communication between processors.

The attenuated boundary terms only need to be computed on and communicated across those boundaries where the radiation leaves each sub-domain (hereafter referred to as downstream boundaries). The corresponding update of the interior of each sub-domain may be carried out afterwards and again is a completely local operation.

The efficiency of our parallelization method depends heavily on how rapidly the attenuated boundary terms may be obtained during the communication step. Due to this dependency, our method is only practical if the radiation is strictly along straight lines and does not diffuse in the transverse direction via interpolation, as in the short characteristics method, for example. This is discussed in detail in Sect. 3.3.

Our technique may be contrasted with other popular approaches to solving the transfer equation in decomposed domains. In the multiple wavefront method (Nakamoto et al. 2001), parallel efficiency is achieved by allowing different ray directions to be treated simultaneously, trying to avoid multiple tasks for the same processor and minimizing the number of idle processors. This method does not impose any restrictions on the radiative transfer scheme, but efficient parallelization requires a large number of ray directions and frequencies to be treated. If a non-diffusive scheme is employed, our technique appears simpler and remains efficient even when the number 
of CPUs greatly exceeds the number of ray directions and frequencies.

\section{The transfer equation}

Radiation couples with the equations of fluid dynamics both through radiative heating and cooling and, if the temperatures are high enough, through radiative pressure. For the applications that we currently have in mind (e.g. stellar convection and protostellar accretion discs), only heating/cooling is important, so $\boldsymbol{\nabla} \cdot \boldsymbol{F}$ enters the energy equation

$\rho \frac{\mathrm{D} e}{\mathrm{D} t}+p \boldsymbol{\nabla} \cdot \boldsymbol{u}+\boldsymbol{\nabla} \cdot \boldsymbol{F}=Q_{\mathrm{diss}}$,

where $\rho$ is the mass density, $p$ is the pressure, $\boldsymbol{u}$ is the fluid velocity, $e$ is the internal energy per unit mass, $\boldsymbol{F}$ is the energy flux, and $Q_{\text {diss }}$ is the (kinetic and/or magnetic) energy dissipation. For radiative energy transfer the energy flux is given by

$\boldsymbol{F}=\oint_{4 \pi} \mathrm{d} \Omega \int_{0}^{\infty} \mathrm{d} v \hat{\boldsymbol{n}} I_{v}(\hat{\boldsymbol{n}})$

where $I$ is the specific intensity giving the amount of energy transported by radiation per unit frequency range per unit area per unit time into a solid angle $\Omega$ in the direction $\hat{\boldsymbol{n}}$.

To determine the specific intensity one has to solve the transfer equation (e.g. Mihalas \& Weibel-Mihalas 1984),

$\hat{\boldsymbol{n}} \cdot \boldsymbol{\nabla} I_{v}=\chi_{v}\left(S_{v}-I_{v}\right)$,

where $\hat{\boldsymbol{n}}$ is the unit vector in the direction of propagation, $\chi_{v}$ is the opacity (per unit volume) or inverse mean free path of a photon, and $S$ is the source function, which gives the ratio between emission and absorption.

The transfer Eq. (3) is here written in its time independent form. This is appropriate for the non-relativistic case, where the maximum fluid velocity is much lower than the speed of light. The flux divergence is then given by

$\boldsymbol{\nabla} \cdot \boldsymbol{F}=\oint_{4 \pi} \mathrm{d} \Omega \int_{0}^{\infty} \mathrm{d} v \chi_{v}\left(S_{v}-I_{v}\right)$.

Following Nordlund (1982), we define $Q_{v}=S_{v}-I_{v}$, giving the cooling rate per ray direction and infinitesimal frequency interval, and the optical depth scale $\tau_{v}=\int \chi_{v} \mathrm{~d} s$, where $s$ is measured along the propagation direction of the ray. It is then possible to rewrite Eq. (3) as

$\frac{\mathrm{d} Q_{v}}{\mathrm{~d} \tau_{v}}=\frac{\mathrm{d} S_{v}}{\mathrm{~d} \tau_{v}}-Q_{v}$

This equation may also be written in integral form,

$Q(\tau)=Q\left(\tau_{0}\right) \mathrm{e}^{\tau_{0}-\tau}+\underbrace{\int_{\tau_{0}}^{\tau} \mathrm{e}^{\tau^{\prime}-\tau} \frac{\mathrm{d} S}{\mathrm{~d} \tau^{\prime}} \mathrm{d} \tau^{\prime}}_{Q^{\text {(intr) }}(\tau)}$,

where the explicit reference to the frequency $v$ has been dropped. By using $Q$ instead of $I$ numerical precision is retained even when the optical depth is very high and $I$ approaches $S$ very closely.
In general the source function $S$ may depend on the intensity itself, i.e. on a nonlocal quantity, turning (6) into an integro-differential equation which has to be solved by means of an iterative scheme, such as Accelerated Lambda Iteration (see Olson et al. 1986). However, during each iteration step the source function is given (i.e. taken from a previous iteration step) and in the following we may - without loss of generality - assume that the source function is independent of intensity.

\section{The radiative transfer scheme}

For the sake of simplicity we here assume that the set of ray directions is chosen in such a way that all rays travel directly through neighboring grid points. This will suffice to motivate our method and we delay the discussion of interpolation schemes for solving the transfer equation for arbitrary ray directions until Sect. 3.3.

With the above assumption, it is in principle easy to solve (5) for all ray directions. Given the cooling rate at a mesh point $n-1$, the discretization of (6) enables us to compute the cooling rate at the next mesh point $n$ in the direction of the ray. Once a given boundary condition is adopted, it is thus possible to determine the cooling rate in the entire simulation box by stepping successively along the ray.

However, in the case of domain decomposition, only those processors adjacent to a boundary of the simulation box are able to immediately compute the correct cooling rate within their sub-domain. All other processors have to wait until they are provided with boundary information from a neighboring processor that already has determined the correct cooling rate. Without further sophistication, this would imply that most computation related to the radiative transfer problem is not carried out in parallel and valuable CPU time is spent in waiting.

Fortunately, for a local source function (e.g. independent of mean intensity), the integral term $Q^{(\mathrm{intr})}$ in (6) represents a valid solution of the transfer equation within each sub-domain, apart only from the contribution from the upstream boundary. We call this the intrinsic solution,

$Q_{n}^{(\mathrm{intr})}=Q_{n-1}^{(\mathrm{intr})} \mathrm{e}^{-\delta \tau_{n-1 / 2}}+\int_{\tau_{n-1}}^{\tau_{n}} \mathrm{e}^{\tau-\tau_{n}} \frac{\mathrm{d} S}{\mathrm{~d} \tau} \mathrm{d} \tau$

with

$Q_{0}^{\text {(intr) }}=0$ and $\delta \tau_{n-1 / 2}=\tau_{n}-\tau_{n-1}$.

The complete solution for an arbitrary boundary condition $Q_{0}$ may be obtained by simply adding the correction term $Q_{0} \mathrm{e}^{\tau_{0}-\tau_{n}}$ to the intrinsic solution on all inner points,

$Q_{n}=Q_{0} \mathrm{e}^{\tau_{0}-\tau_{n}}+Q_{n}^{\text {(intr) }}$.

In order to reduce the idle time of the individual processors we split the calculation of the cooling rate into three distinct parts, two of which may be carried out by all processors in parallel. How this works in detail is illustrated in the following.

\subsection{Non-periodic boundaries}

We first assume that our computational domain is non-periodic in all spatial dimensions. As far as radiative transfer is 
(a)
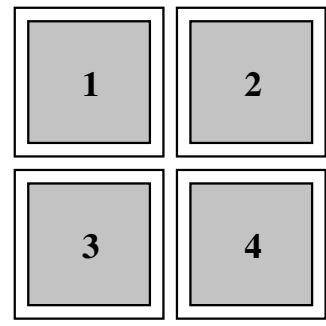

(c)
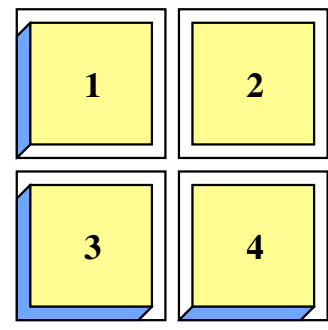

(e)

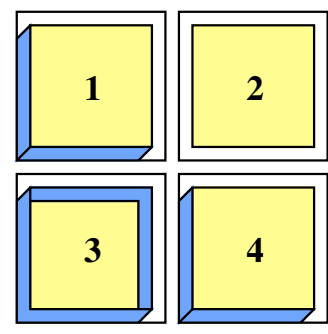

(g)
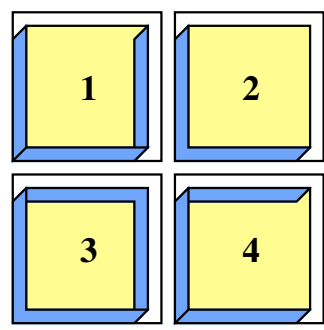

(b)

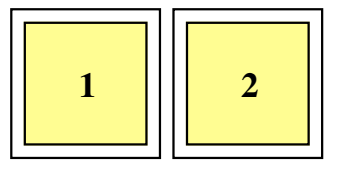

(d)
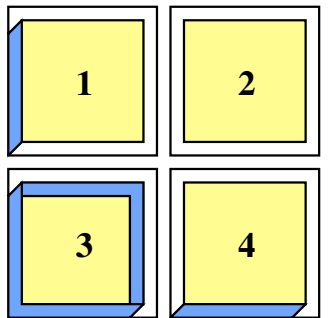

(f)
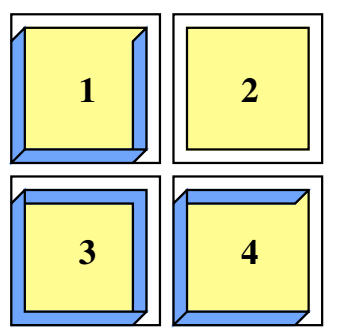

(h)

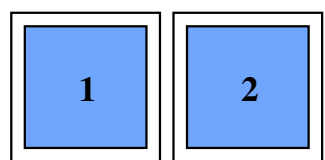

3

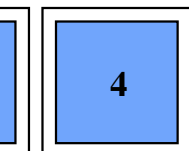

Ray direction: $>$

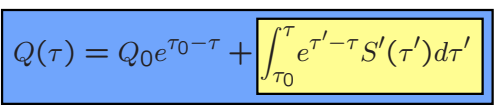

Fig. 1. Illustration of the radiative transfer scheme. See text for details.

concerned, this is the simplest case - the periodic case is treated in the next subsection.

The basic procedure to obtain the solution to the radiative transfer problem in decomposed domains is illustrated in Fig. 1. For the sake of simplicity, the computational domain is two-dimensional and divided into four sub-domains. The transfer equation is solved for rays along the direction indicated. The generalization to three-dimensional domains is trivial.

In the following, $Q_{0}$ refers to the cooling rate on the local upstream boundary of a given sub-domain, which either coincides with the boundary condition of choice for the entire computational domain (global boundary) or overlaps with the downstream boundary of a neighboring processor in the direction opposite to the ray.

The first step is to obtain the intrinsic solution $Q^{(\text {intr) }}$ within each sub-domain, assuming a vanishing cooling rate at the boundary. This corresponds to evaluating the integral in Eq. (6). For each point the cooling rate and the optical depth $\tau-\tau_{0}$ are stored. This step can be carried out by all processors in parallel since no information is required from outside the processor (Fig. 1b).

The communication part follows next. The local boundary cooling rate $Q_{0}$ in the lower ghost zone of processors 3 and 4, as well as in the left ghost zone of processors 1 and 3 are given by the global boundary condition of choice (Fig. 1c). Since all its upstream boundaries are set, processor 3 can immediately compute the correct cooling rate on its upper and right boundaries by adding the correction term $Q_{0} \mathrm{e}^{\tau_{0}-\tau_{N}}$ to the cooling rate obtained from the intrinsic solution (Fig. 1d). Here $\tau_{0}$ and $Q_{0}$ refer to a point in the left (lower) ghost zone and $\tau_{N}$ to the corresponding point at the upper (right) boundary along the ray.

Now that the correct cooling rate on the upper (right) boundary of processor 3 is available, this information is communicated to processor 1 (4) where the boundary condition in the lower (left) ghost zone is set (Fig. 1e). Likewise, processor 1 (4) is now able to compute directly the cooling rate on its right (upper) boundary and can send the values to processor 2 (Figs. 1f and 1g).

In Fig. $1 \mathrm{~g}$ all information necessary to solve the full transfer equation on every point on all processors is available and the communication part is finished. The last step is again carried out by all processors in parallel and independently of each other. It amounts to simply adding the correction term $Q_{0} \mathrm{e}^{\tau_{0}-\tau_{n}}$, this time on all inner points in the sub-domain (Fig. 1h).

\subsection{Periodic boundaries}

For many applications it is convenient to assume periodicity of the simulation box in one or more spatial directions. An example is convection in an infinitely extended plane-parallel layer. While this is trivial to implement for the HD- and MHD-part of a scheme, periodicity introduces a potential difficulty to the radiative transfer scheme.

In the non-periodic case there is always at least one processor where all upstream boundaries are entirely set, once the boundary condition for the whole simulation box has been used. In the example setup of the previous subsection this would be processor 3. By determining the cooling rate on its downstream boundaries and communicating to all its neighbors, all upstream boundaries of these neighbors are entirely set, and so on. This implies that each processor has to propagate boundary values only once through its domain.

In contrast to the above, in the case of periodicity, it might become necessary to propagate boundary values several times through each sub-domain. This is illustrated in Fig. 2. The computational box in this example is taken to be periodic in the horizontal direction, so that only the heating rates in the lower ghost zones of processors 3 and 4 are known a priori from the global boundary condition (Fig. 2b). Without communication, this information does not suffice to entirely cover any of the 
(a)
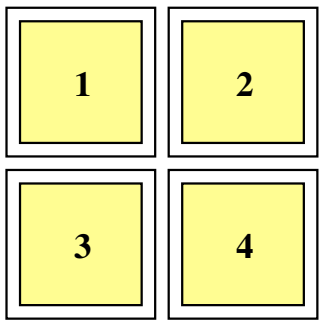

(c)
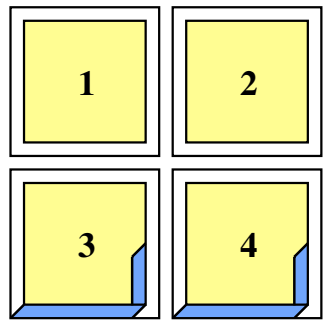

(e)
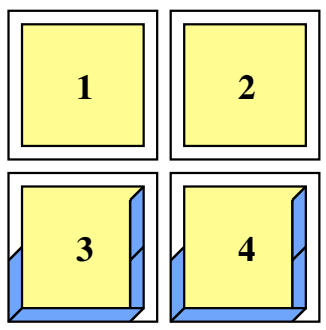

(g)

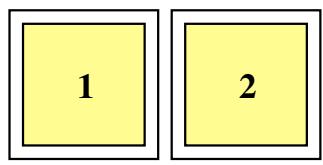

3

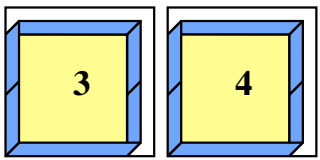

(b)

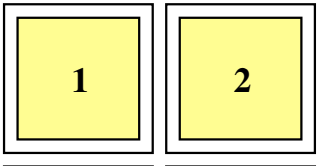

3

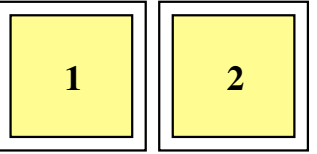

(d)

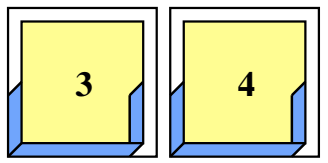

(f)

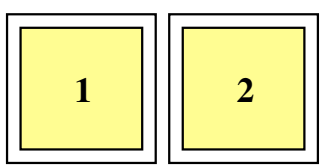

3

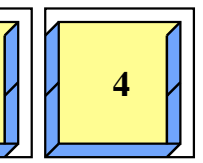

(h)

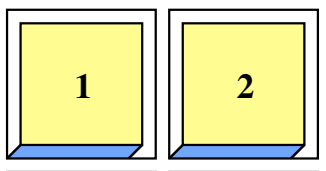

3

4

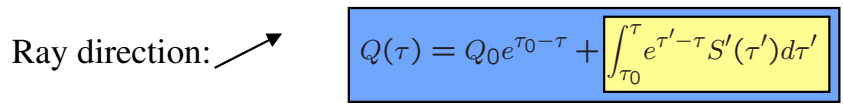

Fig. 2. Same as Fig. 1, except that here the computational domain is periodic in the horizontal direction. Note that the ray travels at an inclination of $22.5^{\circ}$ relative to the horizontal axis.

downstream boundaries of processors 3 or 4 for the given ray direction (Fig. 2c).

In order to cover all up- and downstream boundaries of processors 3 and 4 , it is necessary to communicate the available downstream heating rates along the periodic direction several (in this case two) times (Figs. 2d-2g).

\subsection{Remarks on interpolation}

So far we have only considered rays that pass directly through neighboring grid points. In general we would like to take into account rays with arbitrary inclination, so we have to

interpolate in the angular direction. According to Stone et al. (1992) there are essentially two approaches to this problem which we discuss now briefly, taking into account their compatibility with our proposed parallelization method.

The first approach we consider is to set up exactly one ray per grid point and trace it all the way back to the boundary of the computational domain, interpolating the values for opacity and source function along the way. This is usually called the method of long characteristics. For large numerical grids in two (three) spatial dimensions, the above prescription is, however, hardly ever used in this form as it scales as $N^{3}\left(N^{4}\right)$ with the number of grid points $N$. A common cure for this problem is to introduce in addition to the ordinary hydrodynamical (HD) grid an arbitrarily inclined radiation grid for each ray direction, with grid points along a number of parallel rays in that direction (e.g. Nordlund 1982; Stein \& Nordlund 1988; Razoumov \& Scott 1999; Juvela \& Padoan 2005). The values for opacity and source function are interpolated from the HD grid onto the radiation grid, the transfer equation is solved along each of the parallel rays, and the solution (in terms of the radiative cooling rate) is finally interpolated back onto the HD grid.

This approach is well suited for incorporation into our parallelization method because the radiation does not diffuse out on the radiation grid. Hence, a ray that reaches a point on the downstream boundary of a processor's sub-domain can be traced back to a unique point at the upstream boundary and the attenuated boundary terms may thus be rapidly computed during the communication step of our method. This is crucial for keeping the idle times of the individual processors at a minimum. Furthermore, interpolation between the two separate grids is a completely local operation that can be carried out by all processors in parallel before and after the communication step. Thus, the full advantage of our method can still be exploited. In fact, because it is a local operation, angular interpolation actually improves our method's scaling with the number of processors since the communication time then becomes - relative to the overall expense of obtaining the full solution to the transfer equation - even less significant.

The other approach is to use the method of short characteristics (e.g. Kunasz \& Auer 1988; Auer \& Paletou 1994; Auer et al. 1994). In this method, the rays are cut off at cell boundaries, and the radiation intensity is interpolated onto neighboring grid points. As a result, the radiation along rays that do not travel directly through grid points diffuses away from the exact downstream direction. Due to this diffusion, the radiation reaching one particular grid point on the downstream boundary of a processor's sub-domain depends in a highly non-trivial (unphysical) manner on the radiation coming from a substantial number of grid points on its upstream boundary. In order to propagate the boundary radiation values through downstream processors one would thus essentially have to repeat the radiative transfer solution again, but now with each processor dependent on its upstream neighbor. This would remove the advantage of being able to do most of the work independently on each processor. The short characteristics method is thus not suitable for parallelization with the method presented here. 


\subsection{Periodic rays}

For rays traveling along a periodic direction, there is no boundary condition to start from. However, writing the transfer equation in its integral form,

$Q_{N}=Q_{0} \mathrm{e}^{\tau_{0}-\tau_{N}}+\int_{\tau_{0}}^{\tau_{N}} \mathrm{e}^{\tau-\tau_{N}} \frac{\mathrm{d} S}{\mathrm{~d} \tau} \mathrm{d} \tau$,

where the subscripts 0 and $N$ refer to corresponding points on opposite sides of the simulation box, and using the periodicity condition $Q_{N}=Q_{0}$, it is possible to solve for the cooling rate $Q_{0}$,

$\left(1-\mathrm{e}^{\tau_{0}-\tau_{N}}\right) Q_{0}=\int_{\tau_{0}}^{\tau_{N}} \mathrm{e}^{\tau-\tau_{N}} \frac{\mathrm{d} S}{\mathrm{~d} \tau} \mathrm{d} \tau$.

In a decomposed domain we may thus obtain the full periodic ray solution in much the same way as in the previous section once $Q_{0}$ is known. However, additional communication is required to calculate $Q_{0}$ itself, which means that we have to communicate twice through the simulation box.

The periodic ray solution is illustrated in Fig. 3 for a ray traveling in the positive $x$-direction across 4 processors. The source function and opacity profile are depicted in the two uppermost panels, followed by the intrinsic cooling rate on each processor, the corresponding corrections and the final cooling rate.

\section{Implementation into the Pencil Code}

The method for solving radiative transfer problems, as outlined in the previous section, has been implemented into the PENCIL $\mathrm{CODE}^{1}$. This code has been specifically designed for turbulence simulations in a parallel computing environment using the domain decomposition scheme.

The numerical scheme consists of a third order RungeKutta method due to Williamson (1980) for the time stepping and sixth order centered finite differences in space; see Brandenburg (2003) for details. The code is able to do domain decomposition in two spatial dimensions ( $y$ - and $z$-directions) using the Message Passing Interface (MPI) for interprocessor communications.

For the numerical solution of the transfer equation we approximate the source function by a second order polynomial in optical depth (see Bruls et al. 1999). The integral in Eq. (6) may then be solved exactly. Numerical details are given in Appendix A, available in electronic form. It is however worth noting here that the intrinsic solution where an arbitrary but definite boundary condition is employed may equally well be obtained by virtue of Feautrier's (see Mihalas 1978) or any other suitable method.

In fact, we have found in another context that on most (but not all) CPUs, the Feautrier method is faster by up to about a factor of two, relative to the most optimized integral method (see Appendix A for details). However, since the integral method is more general (applicable for example also in cases with a combination of Doppler effect and polarization in spectral lines), we choose to present its implementation here.

\footnotetext{
${ }^{1}$ http://www.nordita.dk/software/pencil-code
}
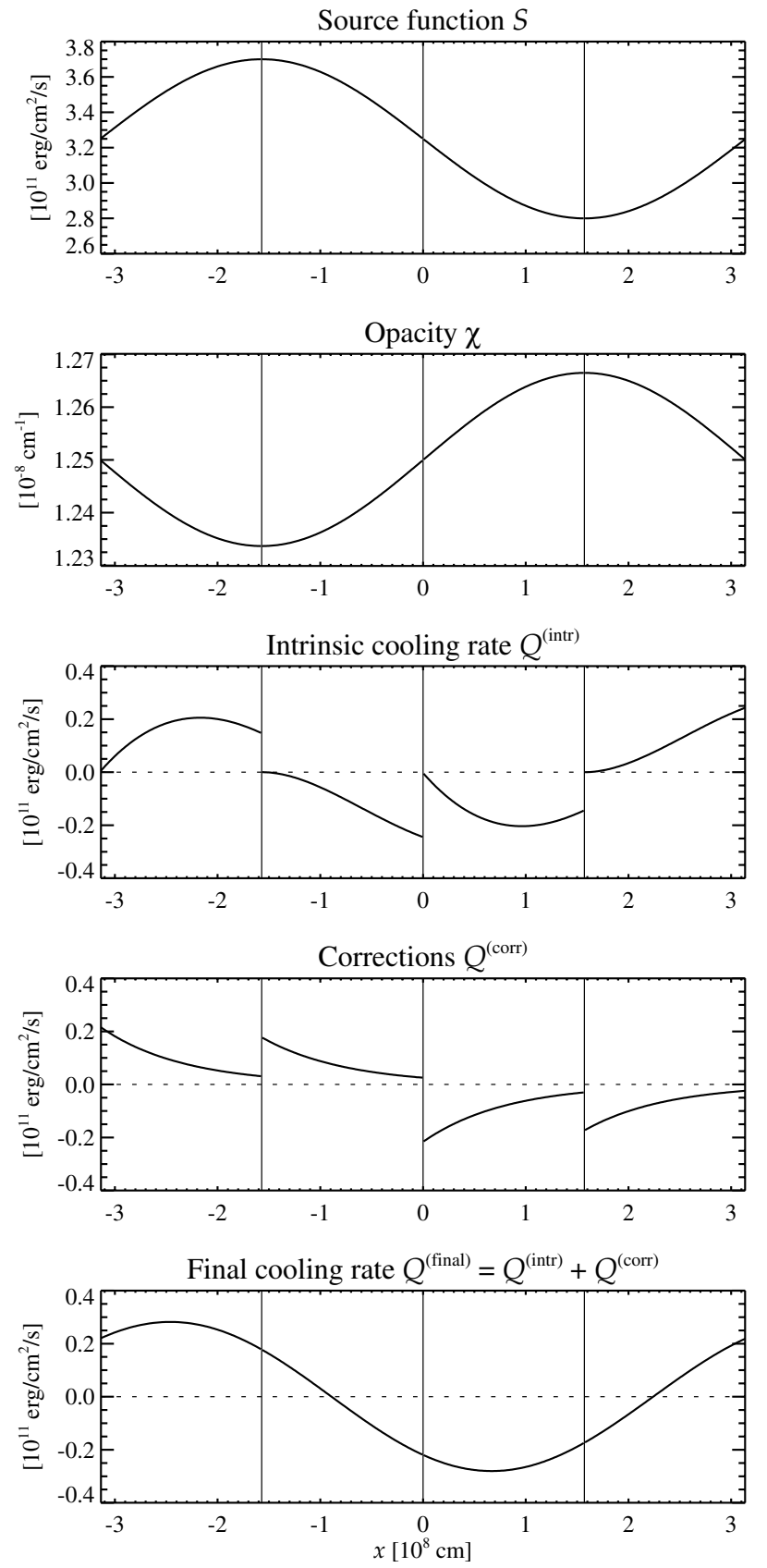

Fig. 3. Illustration of the periodic ray solution for one ray traveling in the positive $x$-direction across 4 processors.

When solving the intrinsic part of the transfer equation we store the difference in optical depth between all grid points and the upstream boundary in a 3-dimensional array. This allows us to quickly compute the attenuated boundary terms and add them to the intrinsic cooling rate on the downstream boundary (during the communication step) as well as to all non-boundary grid-points (afterwards).

\section{Benchmarks results}

The scaling of our method with the number of processors depends on how many processors can simultaneously compute 
the attenuated cooling rates on the downstream boundaries of each processor. This in turn depends on

- the number of ray directions;

- the type of boundary condition (periodic or non-periodic);

- the shape (in terms of grid points) and distribution of subdomains.

To investigate the above dependencies we have performed a series of benchmarks on the Beowulf cluster at the Danish Center for Scientific Computing. All our tests ran on a sub-network with 302 Intel Pentium 4 machines with $3.2 \mathrm{GHz}$ CPUs and 1 GB memory, connected with Gigabit Ethernet and 10-Gigabit uplinks. On the software side, we used the Intel Fortran 8.1 compiler and the LAM MPI implementation.

In the benchmark series the number of processors, $N$, increases from 1 to 128 in powers of two. To be representative of typical applications of our scheme, each benchmark is a shortlived 3-D hydrodynamical simulation of a (gray) solar atmosphere near the surface. As is explained in Appendix B, available in electronic form, the ionization fraction that enters the equation of state is calculated in an iterative fashion from the thermodynamic variables. We use the ionization fraction to calculate the number density of negative hydrogen ions, which are the only source of opacity in these simulations.

To find out whether the choice of boundary conditions for the radiative cooling rate has a significant influence on the scaling, the entire computational domain is either periodic or nonperiodic in the horizontal $x$ - and $y$-directions. The boundary condition in the $z$-direction is in both cases non-periodic.

Furthermore, we have taken into account two different subdomain shapes, characterized by the number of grid points in each spatial direction: "planar" sub-domains with $m_{x}=$ $m_{y}=64$ grid points in the $x$ - and $y$-directions and $m_{z}=$ 32 grid points in the $z$-direction, and "columnar" sub-domains with $m_{x}=m_{z}=64$ grid points in the $x$ - and $z$-directions and $m_{y}=32$ grid points in the $y$-direction. Depending on their shape, these sub-domains are distributed in the following way. If $N$ is an even power of two, there are as many processors in the $y$-direction as there are in the $z$-direction $\left(N_{y}=N_{z}\right)$. If $N$ is an odd power of two, then for planar domains there are twice as many processors in the $z$-direction as there are in the $y$-direction $\left(N_{z}=2 N_{y}\right)$, and for columnar domains it is the other way around $\left(N_{y}=2 N_{z}\right)$. This is illustrated in Table 1 for up to eight processors.

In our present implementation where we only solve for rays that travel through grid points, there are three qualitatively different sets of ray directions determined by the geometry of a grid cell:

- rays along coordinate axes (6 ray directions);

- this plus rays along face diagonals $(6+12-4=14$ ray directions);

- this plus rays along the space diagonals $(14+8=22$ ray directions).

Rays along face diagonals only make up $12-4=8$ additional ray directions since rays along those face diagonals that lie in the horizontal plane are impractical to solve for if periodic boundary conditions are employed, and, for comparison,

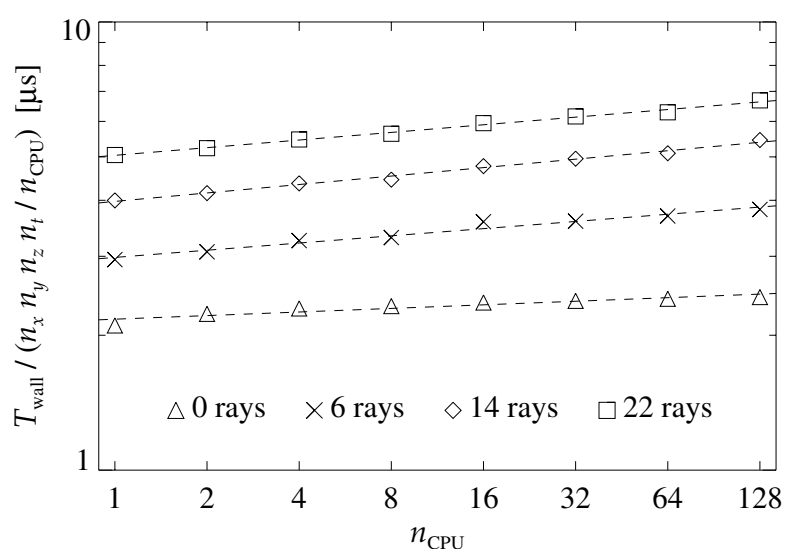

Fig. 4. Wall clock time per grid point and time step in a 3-dimensional simulation of the solar surface for different sets of ray directions - in this case for columnar sub-domains and non-periodic boundary conditions for the cooling rate. The straight lines denote least square fits. Perfect scaling would be represented by a horizontal line.

Table 1. Distribution of sub-domains in $y$ and $z$ for up to 8 processors.

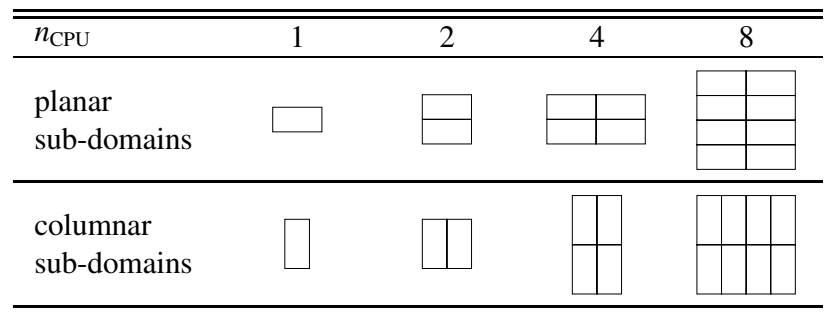

we left them out in the case of non-periodic boundary conditions as well.

The reason we carried out separate benchmarks for each of the above sets is that one could expect a decrease in scaling performance as one goes from 6 ray directions to 22. For rays along the edges of a grid cell there is always a whole layer of processors that can simultaneously receive the boundary cooling rate and propagate the attenuated cooling rate computed at the downstream boundary to the next layer. For inclined rays however, the number of processors that can do the communication simultaneously varies as one communicates through the entire domain and is generally less or equal than for rays along the edges. For comparison with ordinary hydrodynamical domain decomposition, we also included a benchmark with no radiative transfer at all for each type of boundary condition and sub-domain shape. In total, there are thus $4 \times 2 \times 2=16$ benchmarks per processor number.

In Fig. 4 we show the scaling of the wall clock time per grid point and time step in a 3-dimensional simulation of the solar surface for different sets of ray directions using columnar subdomains and non-periodic boundary conditions for the cooling rate. Perfect scaling would be represented by a horizontal line in this figure. Due to the locality of the Navier-Stokes equations, the purely hydrodynamic benchmark series (0 rays) are close to being perfect in the above sense. In comparison, the radiative benchmarks $(6,14$, and 22 rays) show for each type of boundary condition and sub-domain shape a slight increase 


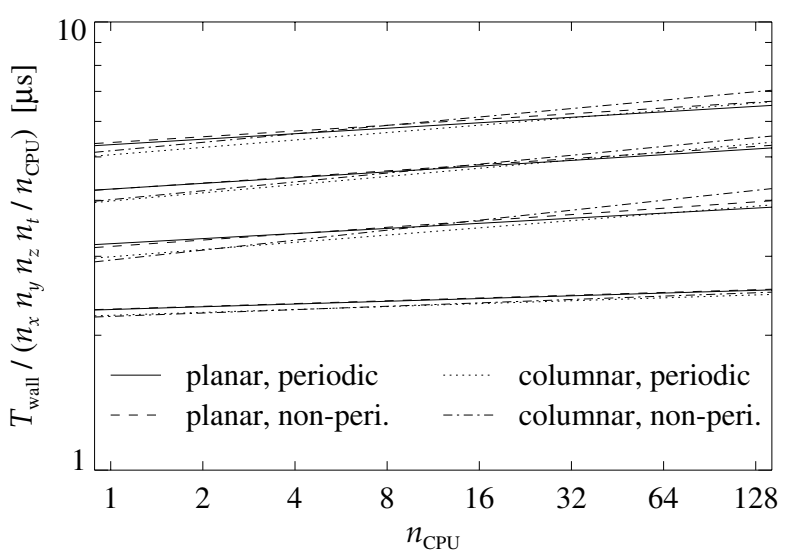

Fig. 5. Least square fits to the wall clock time per grid point and time step for all sets of ray directions, boundary conditions for the cooling rate, and sub-domain shapes.

in the wall clock time with the number of processors, but generally with at most about $30 \%$ as $n_{\mathrm{CPU}}$ varies from 1 to 128 .

In Fig. 5 we compare the scaling behavior for different shapes of the sub-domains, both for periodic and non-periodic rays. The main conclusion to be drawn from this is that the difference in performance is surprisingly small. Nevertheless, as a general trend one can say that the scaling is slightly better for planar than for columnar sub-domains. On the other hand, columnar sub-domains perform slightly better for a small number of processors $\left(n_{\mathrm{CPU}} \leq 8\right)$. Within the accuracy of the measurements, which is not perfect due to constantly varying network and $\mathrm{I} / \mathrm{O}$ performance on the cluster in the course of the benchmark series, we can say that neither the number of ray directions, nor the choice of boundary conditions and subdomain shape has an appreciable impact on scaling with the number of processors.

\section{Conclusions}

In this paper we have presented a parallelization method for carrying out hydrodynamical radiative transfer calculations on massively parallel computers using the domain decomposition scheme. The proposed method is conceptually simple and straightforward to implement. It is also flexible in the sense that the solution of the transfer equation is not limited to the integral method but may also be obtained by direct discretization (Feautrier type methods).

We find that the proposed parallelization method scales almost linearly with inverse number of processors, irrespective of the choice of boundary conditions, sub-domain shape, or number of ray directions. The method is thus ideal for carrying out large hydrodynamical simulations on massively parallel supercomputers using the domain decomposition scheme.

The present implementation into the PENCIL CODE only represents a first proof of concept. The inclusion of more ray directions, a non-uniform vertical mesh, non-gray radiative transfer, radiation pressure, or scattering opacities are all examples of extensions that are still possible within the framework of our parallelization method.
Our focus has not been to optimize the intrinsic part of the radiative transfer calculations, but already in the implementation used for the benchmark series in Sect. 5, one can afford 22 rays per mesh point at a cost of about $40 \%$ of the total time to advance the MHD-equations (60\% relative to advancing only the HD-equations). With the fully optimized integral method (storing and reusing exponentials whenever possible), or with the Feautrier method, one can afford 2 to 4 times more rays per point, depending on the CPU and the type of network.

The number of possible applications of our method is large. It has been tested successfully for simulations of the solar atmosphere where even a gray opacity treatment with a small number of rays already gives quite useful results (but a more accurate model requires an opacity bin coverage, cf. Stein \& Nordlund 1989). To give a list of other applications that is by no means exhaustive, the method is directly applicable to local simulations of accretions discs using the shearing sheet approximations, to global models of stars or discs that are embedded in a Cartesian domain (e.g. Dobler et al. 2006, Freytag et al. 2002), as well as to studies of radiatively driven ionization in HII regions. A generalization to time dependent radiative transfer would allow applications of the method to studies of the reionization of the Universe, as well as to other contexts where effects of the finite speed of light are important.

Acknowledgements. The work of ÅN was supported by grant number 21-01-0557 from the Danish Research Council for Nature and Universe (FNU). Computing time was provided by the Danish Scientific Computing Center (DCSC).

\section{References}

Auer, L. H. 1976, J. Quant. Spectr. Rad. Transf., 16, 931

Auer, L. H., \& Paletou, F. 1994, A\&A, 285, 675

Auer, L. H., Bendicho, P. F., \& Bueno, J. T. 1994, A\&A, 292, 599

Brandenburg, A. 2003, in Advances in nonlinear dynamos (The Fluid Mechanics of Astrophysics and Geophysics, Vol. 9), ed. A. Ferriz-Mas, \& M. Núñez (Taylor \& Francis, London and New York), 269 [arXiv: astro-ph/0109497]

Bruls, J. H. M. J., Vollmöller, P., \& Schüssler, M. 1999, A\&A, 348, 233

Dobler, W., Stix, M., \& Brandenburg, A. 2006, ApJ, 638, 336

Freytag, B., Steffen, M., \& Dorch, B. 2002, AN, 323, 213

Haugen N. E. L., Brandenburg A., \& Dobler W. 2003, ApJ, 597, L141

Juvela, M., \& Padoan, P. 2005, ApJ, 618, 744

Kaneda, Y., Ishihara, T., Yokokawa, M., Itakura, K., \& Uno, A. 2003, Phys. Fluids, 15, L21

Kunasz, P., \& Auer, L. H. 1988, J. Quant. Spectros. Radiat. Transfer, 39,67

Mihalas, D. 1978, Stellar Atmospheres (San Francisco: W. H. Freeman)

Mihalas, D., \& Weibel-Mihalas, B. 1984, Foundations of Radiation Hydrodynamics (Oxford University Press)

Nakamoto, T., Umemura, M., \& Susa, H. 2001, MNRAS, 321, 593

Nordlund, A. 1982, A\&A, 107, 1

Olson, G. L., Auer, L. H., \& Buchler, J. R. 1986, J. Quant. Spectros. Radiat. Transfer, 35, 431

Razoumov, A. O., \& Scott, D. 1999, MNRAS, 309, 287

Stein, R. F., \& Nordlund, A.. 1989, ApJ, 342, L95

Stein, R. F., \& Nordlund, Å. 1998, ApJ, 499, 914

Stone, J. M., Mihalas, D., \& Norman, M. L. 1992, ApJS, 80, 819S

Williamson, J. H. 1980, J. Comp. Phys., 35, 48 
T. Heinemann et al.: Radiative transfer in decomposed domains, Online Material $p 1$

\section{Online Material}




\section{Appendix A: Numerical details}

In order to solve Eq. (6) one uses polynomial approximations for the opacity $\chi$ and the source function $S$. In the current implementation for the PENCIL CoDE, the opacity is assumed to vary linearly from a mesh point $n$ to the next mesh point $n+1$ that lies in the direction of the ray, so the difference in optical depth between these points, calculated by the trapezoidal rule, is

$\delta \tau_{n+1 / 2}=\frac{1}{2}\left(\chi_{n}+\chi_{n+1}\right) \delta s_{n+1 / 2}$,

where $\delta s_{n+1 / 2}$ is the spatial distance between $n$ and $n+1$. A more accurate (but also more expensive) method is based on a cubic spline fit, using the logarithmic derivative of the opacity,

$\delta \tau_{n+1 / 2}=\frac{1}{2}\left[\chi_{n}\left(1+\frac{1}{6} d_{n} \delta s\right)+\chi_{n+1}\left(1-\frac{1}{6} d_{n+1} \delta s\right)\right] \delta s$,

where

$d_{n}=\left(\frac{\mathrm{d} \ln \chi}{\mathrm{d} s}\right)_{n}$,

and, for brevity, we write $\delta s$ instead of $\delta s_{n+1 / 2}$. While theoretically this expression may yield negative values for $\delta \tau$, for reasonably resolved temperature variations $\delta \ln \chi$ should not exceed unity (and certainly must not exceed 6), so in practice this does not happen. A third alternative is

$\mathrm{d} \tau_{n}=\frac{\chi_{n+1}-\chi_{n}}{\ln \left(\chi_{n+1} / \chi_{n}\right)} \mathrm{d} s_{n}$,

but in practice this turns out to be less accurate than Eq. (A.2) above.

To calculate the cooling rate $Q_{n}^{\uparrow}$ due to an 'upwards' directed ray, between $\tau_{n}$ and $\tau_{n+1}$ the quadratic Taylor approximation for the source function is used, i.e. we ignore the third and higher derivatives of $S(\tau)$. Equation (6) then gives

$Q_{n}^{\uparrow}=a_{n-1 / 2} Q_{n-1}+b_{n-1 / 2} S_{n-1}^{\prime}+c_{n-1 / 2} S_{n-1}^{\prime \prime}$

with three coefficients

$a_{n-1 / 2}=\mathrm{e}^{-\delta \tau_{n-1 / 2}}, \quad b_{n-1 / 2}=1-\mathrm{e}^{-\delta \tau_{n-1 / 2}}$,

and

$c_{n-1 / 2}=\mathrm{e}^{-\delta \tau_{n-1 / 2}}\left(1+\delta \tau_{n-1 / 2}\right)-1 ;$

similarly, the 'downwards' directed rays give

$Q_{n}^{\downarrow}=a_{n+1 / 2} Q_{n+1}-b_{n+1 / 2} S_{n+1}^{\prime}+c_{n+1 / 2} S_{n+1}^{\prime \prime}$.

One observes that in the limit of large optical depth, $\left(Q^{\uparrow}+\right.$ $\left.Q^{\downarrow}\right) / 2 \rightarrow-S^{\prime \prime}$ (diffusion limit). This demonstrates that the second derivative $S^{\prime \prime}$ needs to be taken into account, as otherwise the numerically obtained total heating rates would be wrong (they would still be $\propto S^{\prime \prime}$ because up- and down-stream contributions do not cancel exactly, but with an incorrect and resolution-dependent coefficient).

We point out that a factor of nearly two in computational speed may be gained in the radiative transfer part of the computations, at the expense of some storage space, by storing and reusing the $\mathrm{e}^{-\delta \tau_{n-1 / 2}}$ factors between two rays in opposite directions. The speed increase was verified in smaller test cases, but the timings presented in Fig. 4 were obtained without implementing this.

For small values of $\delta \tau$, the expressions for the coefficients $a_{n}, b_{n}$, and $c_{n}$, must be computed in double precision to avoid loss of precision, but the final coefficients may be stored in single precision without noticeable loss of accuracy. On some CPUs further speed-up may be obtained by conditionally using asymptotic expressions for these coefficients, while in other cases, especially where compiler options or special libraries are available to enable vectorization or other optimization of exponentials, it may be faster to retain the explicit computation of exponentials. We have implemented coding that automatically chooses between these two alternatives, based on an initial comparison of the speeds.

The derivatives of $S$ with respect to the optical depth $\tau$ have to be computed on an irregularly spaced grid, i.e.

$S_{n}^{\prime}=\left(\frac{\delta S_{n-1 / 2}}{\delta \tau_{n-1 / 2}} \frac{\delta \tau_{n+1 / 2}}{2}+\frac{\delta S_{n+1 / 2}}{\delta \tau_{n+1 / 2}} \frac{\delta \tau_{n-1 / 2}}{2}\right) / \overline{\delta \tau_{n}}$,

$S_{n}^{\prime \prime}=\left(\frac{\delta S_{n+1 / 2}}{\delta \tau_{n+1 / 2}}-\frac{\delta S_{n-1 / 2}}{\delta \tau_{n-1 / 2}}\right) / \overline{\delta \tau_{n}}$,

where $\overline{\delta \tau_{n}}=\left(\delta \tau_{n-1 / 2}+\delta \tau_{n+1 / 2}\right) / 2$ (see Bruls et al. 1999). The procedure to compute radiative energy transport in Cartesian geometry is then straightforward. For each ray direction there are in three dimensions - dependent on the inclination - either one, two or three upstream boundaries where a certain boundary condition is employed and the iteration as defined by (A.5) starts. By moving stepwise through the entire box it is possible to determine the cooling rate on every single mesh point and for all desired ray directions.

\section{Appendix B: Simulations of the solar atmosphere}

The benchmark we used in Sect. 4 is a short-lived simulation of the solar atmosphere near the surface without magnetic fields. For all benchmarks in the series (see Sect. 5) the physical size of the simulation box is $6 \mathrm{Mm} \times 6 \mathrm{Mm} \times 6 \mathrm{Mm}$ in $x, y$, and $z$ respectively. To account for the varying resolution of the numerical grid during the benchmark series, the viscosity is appropriately adjusted. The simulation box is periodic in both $x$ and $y$, even if the boundary condition for the radiative cooling rate is non-periodic as it is the case for half of the benchmark runs.

Furthermore, we use an equation of state that accounts for hydrogen ionization but ignores the negative hydrogen ion, $\mathrm{H}^{-}$, and hydrogen molecule formation. The hydrogen ionization fraction is obtained iteratively from entropy and density by solving Saha's equation. The $\mathrm{H}^{-}$opacity is then calculated from the number density of $\mathrm{H}^{-}$, which is again calculated from the number density of electrons and neutral hydrogen. The number density of $\mathrm{H}^{-}$is very small, so even though $\mathrm{H}^{-}$is the most important (and, in this simulation, only) contributor to the opacity, ignoring it in the equation of state is justified. 